\section{CHANGES IN DIGNITY AND RESPECT AT THE END OF LIFE: CROSS-SECTIONAL ANALYSIS OF DATA FROM THE VOICES BEREAVEMENT SURVEY (2011-2015)}

Benjamin Allison, Preeti Sanhu, Katherine Sleeman. The Cicely Saunders institute, King's College London

\subsection{6/bmjspcare-2019-ASP.89}

Background An aim of the National End of Life Care Strategy (2008) was to improve dignity and respect in end of life care (EoLC). The VOICES survey was commissioned in 2011 as the first national post-bereavement postal survey, a key component of which was to assess dignity and respect at the end of life. The aim of this study was to explore changes in dignity and respect perceived by bereaved relatives over the fiveyear period that the VOICES survey was commissioned.

Methods Aggregate data from VOICES post-bereavement surveys (2011-2015) was obtained from the Office of National Statistics. Information about dignity and respect was extracted and dichotomised into satisfied ('always'and 'most of the time') and unsatisfied ('some of the time'and 'never'). A chisquared test for trend was used to analyse changes over time in dignity and respect, for each of seven categories of health care professionals.

Results There were 107206 responses to the VOICES surveys over 5 years (average response rate 44.4\%). Improvements in perceived dignity and respect from 2011 to 2015 were found with respect to five categories of health care professional: GPs ( $0.7 \%$ improvement in satisfaction to $60.9 \%, \mathrm{p}=0.016)$; hospital doctors $(1.7 \%$ improvement to $85.0 \%, \mathrm{p}=0.0036)$; hospital nurses $(5.2 \%$ improvement to $80.6 \%, \mathrm{p}<0.0001)$; hospice doctors $(0.8 \%$ improvement to $95.5 \%, \mathrm{p}=0.0001)$; hospice nurses $(1.9 \%$ improvement to $95.2 \%, \mathrm{p}=0.0001)$. District/ community nurses showed a $1.4 \%$ decrease in satisfaction to $92.6 \% \quad(\mathrm{p}<0.0001)$. Care home staff showed no change $(\mathrm{p}=0.2)$.

Conclusions The data has demonstrated a general trend of improvement with regard to dignity and respect experienced from healthcare professionals. District/community nurses showed a decrease in dignity and respect however, this could be attributed to a regression to the mean or due to their high baseline, therefore this would not be cause for concern. The low satisfaction demonstrated with GPs should be investigated.

\section{THE GOLD STANDARDS FRAMEWORK FOR IMPROVING THE QUALITY OF END-OF-LIFE CARE IN THE COMMUNITY: A SYSTEMATIC REVIEW AND QUALITATIVE SYNTHESIS}

Preeti Sandhu, Benjamin Allison, Katherine E Sleeman. King's College London, Cicely Saunders Institute of Palliative Care Policy and Rehabilitation

\subsection{6/bmjspcare-2019-ASP.90}

Background The Gold Standards Framework (GSF) was established in 2000 to help ensure delivery of high-quality end-oflife care within general practices, and has since been adapted for care homes and hospitals. The GSF has been endorsed by the UK Department of Health, with a national roll-out seeing all UK general practices and $25 \%$ of care homes adopting the GSF in some form. The evidence base for the GSF in the community has not been systematically evaluated. The aim of this study was to synthesise the qualitative evidence for the GSF on improving end-of-life care in the community.
Method A systematic literature review of published research from 2000 to 2018 using Medline and PubMed databases, in line with PRISMA guidelines. A thematic matrix analyses approach was adopted. Studies were quality assessed using the QualSyt tool.

Results Of the 115 studies identified, 19 studies reported on qualitative findings of the GSF implementation in the community setting. All 19 studies collected data from staff members and 4 studies also included patients and/or relatives. The GSF was felt to facilitate improved written and verbal communication in both nursing care homes and primary care teams, and to enhance methods of teamworking. 16 of 19 studies reported increased uptake of a palliative care register to identify end-of-life patients. Barriers to use of the GSF included a lack of sustainability due to high staff turnover, as well as an increased workload and difficulties in discussing end-of-life care with relatives.

Conclusions These findings support the use of the GSF in improving the quality of end-of-life care through collaborative working and increased communication across healthcare teams. Further studies are required to understand attitudes of patients and relatives. Challenges for policy makers include ensuring end-of-life care tools are both sustainable and flexible according to local population and workforce needs.

\section{Ethics | Posters $68-75$}

\section{WHY ARE PATIENTS NOT MORE INVOLVED IN THEIR OWN SAFETY? A QUESTIONNAIRE-BASED SURVEY IN A MULTI-ETHNIC NORTH LONDON POPULATION}

Zouina Assassi, Iman Ahmedani, Wai Yoong, Rhama Abdinasir, Michael Adofo Kwakye, Harriet Taylor, Danya Chandrakumar, Maud Nauta, Max Denning. University College London, North Middlesex University Hospital, Camden Health Improvement Practice

\subsection{6/bmjspcare-2019-ASP.91}

Background An estimated 850000 cases of unintentional medical injuries occur every year, at least half of which are thought to be preventable. Patients receiving palliative care are particularly vulnerable to medical errors and their consequences. Patient participation in their own safety has welldemonstrated benefits in reducing errors. Understanding how patients feel about participating in their own safety and how to improve patient engagement could help reduce medical errors in palliative care. The authors sought to examine patient participation in a range of safety-related behaviours and investigate how they varied with different patient demographics.

Methods A 20-point questionnaire was employed exploring safety-related behaviours, particularly looking at patient willingness to challenge healthcare professionals and to notify them of potential errors. Data on sex, age, ethnicity, English language proficiency, duration in the United Kingdom, employment and education status was also collected.

Results 195 patients were invited to participate in this study and 175 patients completed the questionnaire. Female patients who had tertiary education, those who were fluent in English and under the age of 60 years were statistically more likely to feel responsible for their own safety and take an active role in their safety. Older male patients of lower education status are statistically less likely to question staff on hand hygiene. 